\title{
L'ECHINOCOCCOSE EN ISLANDE. \\ SUR LE MODE DE CONTAMINATION HUMAINE
}

\author{
Par Matthias EINARSSON
}

(de Reykjavik)

Il y a soixante ans, H. Krabbe et Jón Finsen suivirent l'évolution du Tænia echinococcus en Islande. Ayant fait ingérer des kystes hydatiques humains à de jeunes chiens, ils sacrifièret ces animaux quelques mois plus tard, et chez quelques-uns d'entre-eux, ils trouvèrent des ténias échinocoques (1). Ils firent ensuite absorber à un agneau les œufs des ténias obtenus et, trois mois plus tard, quand l'animal fut sacrifié, son foie et ses poumons renfermaient de très petits kystes.

Les mêmes expérimentateurs essayèrent de contaminer des chats, de la même façon, mais sans succès. Depuis lors, il a été prouvé que le chat est loin d'être aussi susceptible que le chien de devenir l'hôte de $T$. echinococcus. Pourtant F. Dévé a réussi à obtenir des ténias échinocoques dans l'intestin d'un chat auquel il avait fait absorber des kystes fertiles. D'autres auteurs ont fait la même constatation. Mais ces cas sont très rares.

En Islande, il n'existe pas d'autres animaux qu'on puisse supposer capables d'héberger le ténia échinocoque. Bien que le renard soit du même genre que le chien, le parasite en question n'a jamais été rencontré chez lui. A vrai dire, cette recherche n'a pas été faite dans notre pays.

Les relations quotidiennes entre les hommes et les chiens et chats, en Islande, ont toujours été très intimes et elles le restent encore. Ces animaux sont très nombreux. Pour ce qui est des chats, j'en ignore le nombre et d'ailleurs cela importe peu, car ils n'ont pas la même voracité que les chiens pour la viande crue; et d'autre part, nous avons vu que le ténia échinocoque se développe difficilement chez eux.

En 1863, lorsque $\mathrm{H}$. Krabbe séjournait en Islande, la population n'était que de 70.000 habitants, tandis que le nombre des chiens

(1) Toutes les précautions avaient êté prises pour que les chiens ne puissent se procurer ailleurs une nourriture pouvant contenir des kystes hydatiques. Une preuve en est qu'on ne trouva, chez les animaux infestés, ni $T$. marginata, ni T. conurus.

Annales de Parasitologie, T. IV, $\mathrm{N}^{\circ} 2 .-1^{\text {er }}$ avril 1926, p. 172-184. 
était estimé de 15 à 20.000 : soit un peu plus de 20 chiens pour 100 habitants. Depuis lors, le nombre des chiens a diminué, pendant que la population s'est accrue. En 1922, on comptait 93.000 habitants et seulement 6.700 chiens: soit un peu moins de 7 chiens pour 100 habitants.

H. Krabbe avait autopsié 100 chiens islandais. Chez 28 d'entreeux, il trouva des $T$. echinococcus, c'est-à-dire environ chez le quart des animaux. Il constata, en outre, la présence d'autres ténias : chez $750 / 0, T$. marginata (provenant des cysticerques de l'épiploon du mouton : $C$. tenuicollis) et chez $180 / 0, T$. cœnurus (qui cause le cénure du cerveau des moutons : Conurus cerebralis).

Au premier abord, il paraît assez surprenant que le quart seulement des chiens aient été infestés de ténias échinocoques. Car, à cette époque, les chiens avaient de nombreuses occasions de dévorer des échinocoques. Mais en examinant les faits de plus près, en comparant la fréquence respective de $T$. marginata, $T$. echinococcus et $T$.' cœnurus, les proportions semblent bien répondre à la facilité que les chiens pouvaient avoir de se procurer les différentes espèces.

En effet, quand on abattait les moutons, il était d'usage d'extraire les cysticerques de l'épiploon en enlevant les viscères. Ces cysticerques étaient jetés aux chiens qui les dévoraient aussitôt. Au contraire, les chiens ne pouvaient accéder avec autant de facilité aux hydatides du foie et du poumon que lorsque les organes étaient tellement parasités qu'ils étaient tout à fait impropres à l'alimentation. Quant aux cénures du cerveau, les chiens avaient encore plus de difficultés à les atteindre. Ce ne pouvait être qu'après quelques jours, quand les têtes de moutons avaient été préparées ; mais à ce moment beaucoup de parasites étaient sans doute déjà morts. Il en était de même dans le cas où les chiens mangeaient des charognes contaminées.

Malgré tout, on aurait cru que les chiens eussent dû être infestés de ténias échinocoques dans une plus grande proportion. Toutefois, il ne faut pas oublier que la vie des ténias est relativement courte. Or, en Islande, on ne tue les moutons qu'à une seule époque : de la mi-septembre à la mi-octobre. C'est la seule période de l'année où les chiens ont l'occasion de se contaminer. $\mathrm{H}$. Krabbe a séjourné en Islande de mai à octobre 1863 : tous les ténias trouvés par lui étaient donc ceux de l'automne précédent. Aussi peut-on supposer que son pourcentage était inférieur à la réaljté, bien que des ténias n'aient pas dû nécessairement se développer chez tout chien ayant mangé un kyste hydatique, même vivant et fertile, ainsi qu'il résulte de l'expérience de Krabbe et Finsen. 
Depuis le séjour de Krabbe en Islande, aucune recherche sur l'échinococcose des animaux n'avait été faite dans ce pays jusqu'à l'automne dernier, quand le gouvernement, à mon instigation et après avoir consulté le chef vétérinaire Magnus Einarsson, chargea les vétérinaires et deux médecins cantonaux de faire le relevé des échinocoques du foie et du poumon chez les moutons abattus dans leurs districts respectifs. En Islande, on ne compte que 4 vétérinaires. En sorte qu'ils ne pouvaient, à eux-seuls, examiner tous les moutons abattus, surtout étant donné qu'on tue les moutons partout en même temps (septembre-octobre). Les médecins cantonaux sont chargés de la vérification et de l'estampillage légal de la viande dans les localités privées de vétérinaires.

Le résultat de cetfe statistique a été le suivant :

\begin{tabular}{|c|c|c|c|}
\hline Observateur & LIEU D'BXAMEN & $\begin{array}{l}\text { NOMBRE } \\
\text { DE MOUTONS } \\
\text { ABATTUS }\end{array}$ & $\begin{array}{l}\text { Proportion } \\
\text { D'ANIMAUX } \\
\text { ATtEINTS } \\
\text { D'ÉCHINoCOCCOSE }\end{array}$ \\
\hline $\begin{array}{l}\text { Magnus Einarsson, chef vé- } \\
\text { térinaire. . } \\
\text { Hannes Jonsson, vétérinaire } \\
\text { Arni Arnason, médecin . } \\
\text { Jonas Kristjansson, médecin } \\
\text { S. Hlidar, vétérinaire. } \\
\text { J. Palsson, vétérinaire. } \\
\text { Toraux. }\end{array}$ & $\begin{array}{c}\text { Reykjavik } \\
\text { Borgarnes } \\
\text { Budardalur } \\
\text { Saudarkrokur } \\
\text { Akureyri } \\
\text { Reydarfjördur }\end{array}$ & $\begin{array}{l}1.915 \\
2.029 \\
1.115 \\
3.530 \\
7.140 \\
1.695 \\
17.424\end{array}$ & $\begin{array}{c}3,29 \% \\
26,1- \\
17,9- \\
11,7- \\
12,6- \\
2,77- \\
12,42 \%\end{array}$ \\
\hline
\end{tabular}

On remarque de grandes différences dans les pourcentages qui vont de $26,10 / 0$ à $2,770 / 0$. Mais on doit tenir compte de ce fait que les chiffres en question concernent deux extrémités de l'Islande.

Il est depuis longtemps reconnu que sur la côté Est de l'île, les kystes hydatiques sont très rares chez le bétail aussi bien que chez l'homme. Cette différence ne me surprend pas, car il est probable que la majorité des moutons sont ceux des fiords, qui se nourrissent, sur les plages, de varech lavé par la mer et naturellement exempt d'œufs de ténias. Ce qui m'étonne beaucoup plus, c'est la grande différence relevée entre les moutons de Borganes et ceux de Reykjavik (26,1 $0 / 0$ et $3,290 / 0)$, étant donné qu'il s'agit d'animaux appartenant à deux district voisins. Selon moi, le nombre de 
moutons examinés dans ces deux endroits a été trop faible et a dû donner, par suite, un résultat inexact. Sans doute aurait-on un résultat plus exact en groupant les moutons abattus sur la côte Sud de l'Islande (Reykjavik, Borgarnes et Budardalur) : au total 3.059 moutons avec un pourcentage de contamination échinococcique de $15,90 / 0$. Le Nord du pays (Saudarkrokur, Akureyri) donnerait un total de 10.670 moutons abattus, avec un pourcentage d'infestation un peu moins élevé : $12,290 / 0$.

Chez les moutons parasités on a trouvé presque uniquement des kystes du foie et très peu de kystes du poumon. Pourtant je dois noter, à cet égard, une grande différence assez surprenante : des kystes du poumon ont été constatés chez 41 sur 47 des moutons atteints d'échinocoques, à Reydarfjödur (côte Est), tandis que sur 453 moutons parasités observés à Borgarnes (côte Sud-Ouest), 4 seulement étaient atteints de kystes du poumon. C'est un fait très singulier dont la cause m'échappe.

Au total, 17.424 moutons ont été examinés, ce qui représente $3,20 / 0$ de tous les moutons islandais. En effet, le total était, en 1922 , de 550.000 moutons. Sur ce nombre, $12,420 / 0$ étaient atteints de kystes échinococciques (1).

Le nombre des chiens porteurs de ténias échinocoques n'a pu être établi, faute de recherches nouvelles à ce point de vue. Mais il est permis de supposer que la proportion entre $T$. echinococcus et $T$. marginata doit être restée sensiblement la même qu'à l'époque du séjour de Krabbe en Islande. Cet observateur avait trouvé un chien porteur du premier parasite, pour trois porteurs du second $(280 / 0$ et $710 / 0)$. A l'heure actuelle, J. Kristjansson compte un mouton atteint de kystes hydatiques pour trois atteints de cysticerques $(11,70 / 0$ et $31,50 / 0)$. Ces chiffres confirment mon hypothèse.

$$
* *
$$

Selon E.-J.-Mc. Donnel, l'antique " cave canem " devrait être ia devise des Australiens. Cette devise serait tout aussi applicable à l'Islande, s'il est vrai, comme Krabbe le disait en 1863, qu'il y ait des milliers d'œufs de ténias dans les excréments d'un chien sur quatre. Néanmoins, il faut tenir compte d'autres facteurs. Car, s'il

(1) Ces chiffres concernent les seuls moutons adultes, et on n'a tenu compte que des kystes visibles et palpables.

En Islande, les échinocoques, les cysticerques et les cénures sont vulgairement appelês du même nom : " sullur ", qui signifie "vessie d'eau " (boule d'eau).

Le Dr J. Kristjansson, de Saudarkrokur, a fait relever spécialement les cysticerques. On en a trouvé chez $300 / 0$ des moutons; ainsi $400 \%$ des moutons islandais seraient atteints de « vessies d'eau ». 
est incontestable que le chien est la source de la maladie, l'œuf du ténia fait des détours sur le trajet qui va de l'intestin du chien au tube digestif de l'homme. La contamination hydatique humaine présente, en effet, beaucoup de particularités étranges.

Dès les premiers temps, on a remarqué, en Islande, que les femmes étaient beaucoup plus souvent intéressées que l'homme par la maladie hydatique, le contraste étant surtout frappant entre 20 et 40 ans (Finsen). Le fait a été confirmé ultérieurement par Jonassen. Et la même proportion s'est maintenue jusqu'à ces dernières années, époque à laquelle s'est produit un important changement sur lequel nous reviendrons plus loin. L'atteinte prédominante des femmes par la maladie hydatique s'observe également en Tunisie. Par contre, en Australie et en Argentine, le nombre des hommes contaminés est plus élevé que celui des femmes.

Depuis les études de Krabbe et de Finsen sur l'échinococcose en Islande, il n'y a eu, dans ce pays, aucune divergence de vues sur le mode de contamination humaine. Jonassen exprime une opinion semblable à celle des deux auteurs précédents, mais il a suivi de façon plus minutieuse le processus d'infestation. Voici ce qu'il écrit à ce sujet :

"Il n'est pas douteux que les moutons se contaminent dans les pâturages ou en mangeant du foin. Toutefois, il est à remarquer que les brebis (et les vaches) qui.paissent près des fermes s'infestent beaucoup plus facilement que les moutons (et les bœufs) qui passent l'été dans les montagnes. Quant aux gens, c'est probablement dans leurs maisons qu'ils se contaminent. C'est ce qui semble ressortir du fait que le nombre des femmes atteintes est beaucoup plus élevé que celui des hommes.

"Les chiens restent beaucoup dans les maisons, dans la cuisine et les appartements, le jour aussi bien que la nuit. Ils se frottent contre la vaisselle, lappent l'eau des seaux, lèchent les assiettes, etc. Ils abandonnent même des anneaux de ténias sur le plancher ou au contact des ustensiles de cuisine. Ces anneaux se dessècheront et leurs œufs voleront avec le reste de la poussière. En outre, les chiens lèchent les mains et la figure des enfants, aussi bien que celle des adultes. Les femmes font la cuisine, lavent la vaisselle, balaient l'appartement et restent ordinairement à la maison dans cette athmosphère empestée. C'est pourquoi elles sont plus exposées à la contamination. "

Les savants étrangers partagent à peu près le même avis. Par exemple, R.-V. Neumann et Martin Mayer : "Les chiens laissent continuellement échapper des anneaux et des œufs de ténias dans 
leurs excréments, et lorsque le chien se lèche, les œufs se trouvent disséminés sur son corps et surtout sur son museau. De là, ces œufs continueront leur chemin jusqu'aux vêtements, à la peau et aux aliments des personnes. Et ainsi ils parviendront finalement dans le tube digestif humain ". Herxheimer et Kaufmann expriment la même opinion. J.-Mc. Donnell croit qu'en Australie les gens se contaminent principalement par l'eau de boisson polluée par les ehiens.

En revanche, F. Dévé écrit (in "Enquête étiologique sur l'échinococcose en Tunisie ", Archives de l'I. P. de Tunis, déc. 1923): "Contrairement à ce qui se répète partout, nous ne sommes pas persuadé que le chien soit particulièrement dangereux par sa langue et par sa bave. La gueule et même le museau du chien ne sont sans doute qu'exceptionnellement et très passagèrement porteurs de germes hydatiques. Pour notre part, nous pensons que ce n'est pas en lapant l'eau d'un récipient, en lèchant les ustensiles de cuisine, la vaisselle, les mains ou même la figure de ses maîtres que le chien risque surtout de les contaminer. Non pas que nous ne condamnions, bien entendu, ces fâcheuses habitudes! Mais nous estimons le "fidèle ami de l'homme " autrement redoutable pour celui-ci par ses pattes sales et par son poil poussiéreux et souillé. Souillé non seulement dans la région anale, mais sur tout le corps, du fait que l'animal se couche sur le sol qu'il a infesté. Bref, si l'homme se contamine au contact direct de son chien, c'est sans doute en le caressant plus qu'en se laissant lécher par lui. Mais, d'une façon générale, le chien ténifère est probablement moins souvent dangereux par son contact direct, possible à éviter, que par son voisinage inévitable ».

Dévé pense, de plus, qu'en Tunisie, la géophagie des enfants intervienț pour une part et aussi les légumes consommés crus par les Arabes, de même que l'eau de boisson provenant de puits ou d'eaux de surfaces polluées. Les femmes arabes se contamineraient plus fréquemment, du fait d'un voisinage plus étroit avec les chiens dans les maisons et dans les tentes, - d'une façon qui ressemble, en somme, à celle qui a été décrite en Islande par J. Jonassen.

"Maladie des mains sales " (Dévé); " obrifaveiki " (isl., G. Claessen) ; " filthdisease " (angl.) : telles sont les qualifications attribuées à l'échinococcose. Tous ces termes sont assez bons; le premier est très frappant.

En Islande, on a remarqué depuis longtemps que la maladie est plus rare dans les villages maritimes qu'à la campagne (J. Finsen). Pourtant, les chiens de ces agglomérations-là ont un accès aussi 
facile à la nourriture infestante que leurs camarades de la campagne ; car on y tue également des moutons tous les automnes, et avec aussi peu de précautions quant aux viscères parasités. Les chiens $\mathrm{y}$ vivent dans une intimité aussi étroite avec les habitants, et la propreté a toujours été regardée comme y étant un peu inférieure à celle de la campagne. En outre, les sources de ces villages maritimes sont encore plus exposées aux souillures que les ruisseaux de la campagne. En sorte que si le chemin de la contamination allait directement du chien à l'homme, la maladie hydatique devrait être presque aussi générale dans les agglomérations cotières que dans les villages de l'intérieur, surtout si l'on remarque qu'un chien infesté devrait toucher un plus grand nombre de personnes dans une population plus dense.

Ces considérations indiquent qu'il doit exister, dans le processus d'infestation, quelque étape intermédiaire qui a échappé jusqu'à ce jour. Car les explications données par J. Finsen, J. Jonassen et par F. Dévé du phénomène remarquable de la prédominance de l'atteinte des femmes sont manifestement insuffisantes. Elles n'expliquent pas davantage pourquoi la différence d'atteinte des deux sexes est plus marquée à une certaine période de l'existence.

Nous ferons tout d'abord observer qu'en Islande les chiens ne déposent pas leurs excréments dans les maisons, mais hors des maisons. De sorte que ces matières fécales ténifères devraient surtout menacer ceux qui travaillent dehors, c'est-à-dire les hommes. Lorsque, indépendamment de tout excrément, les chiens laissent échapper des anneaux de ténias dans les maisons, ce ne sont pas des anneaux de $T$. echinococcus, mais bien des anneaux de $T$. marginata ou de $T$. cœnurus, lesquels n'ont rien à voir avec l'échinococcose humaine (1).

En admettant que le facteur le plus dangereux soit le séjour des chiens dans les maisons (souillure des ustensiles de cuisine, de l'eau, etc.), le danger devrait être le même pour l'homme que pour la femme, car l'un et l'autre mangent la même nourriture et boivent la même eau.

Il semble que devraient être surtout exposés les jeunes enfants, qui se traînent sur le plancher, jouent avec.les chiens et ne se

(1) T. echinococcus mesure seulement 4 à $6 \mathrm{~mm}$ de longueur et son siège est le duodénum. L'anneau qui se détache a donc de 2 à 3 mèires à parcourir avant d'arriver à l'extérieur. Il est tout à fait impossible qu'il accomplisse cette traversée intestinale jusqu'à l'anus sans être dissous et mélangé aux matières fécales. Au contraire, $T$. marginata peut atteindre une longueur de 3 mètres et $T$. cœenurus de $0 \mathrm{~m}$. 50, ce qui rend très compréhensible l'issue d'anneaux complets et isolés. Mais, encore une fois, les anneaux en question sont sans aucun rapport avec la maladie hydatique humaine. 
soucient ni de propreté, ni de danger. Or, l'infestation des enfants ne paraît pas être fréquente en Islande. Bien que la croissance des kystes hydatiques soit lente, il est à supposer que, parmi les kystes qui deviennent apparents de 20 à 40 ans, ce n'est qu'une minorité qui aura pris naissance dans la première enfance du malade. Aussi bien, la différence de fréquence de la maladie entre les hommes et les femmes dans la période qui s'étend de 20 à 40 ans, serait plus inexplicable encore si la contamination remontait à l'enfance.

Il est certain que le sexe par lui-même ne joue aucun rôle, comme le montre bien ce fait que, en Australie et en Argentine, chez des individus de notre propre race, la proportion se trouve inversée, les hommes formant la majorité des individus atteints. Enfin si, depuis le temps de Finsen et de Jonassen, la manière de vivre s'est améliorée en Islande, si la propreté a fait des progrès, le changement a intéressé femmes comme hommes, et enfants comme adultes.

C'est donc ailleurs qu'il faut chercher la raison de la différence extrêmement marquée qui existe entre le nombre d'hommes et de femmes affectés par l'échinococcose.

Le chien abandonne ses excréments chargés d'œufs de ténias échinocoques quelque part dans l'herbe. Desséchées et pulvérisées, ces matières fécales se trouveront disséminées dans les pâturages par le vent, la pluie ou entraînées par l'eau courante. Les œufs du ténia échinocoque offrent une grande vitalité : ils supportent la dessiccation et une chaleur assez élevée pendant 10 et 11 jours ; ils peuvent vivre 16 jours dans l'eau et 4 mois dans la glace (Dévé). De là on peut conclure que, pour ce qui concerne l'Islande, les œufs en question pourront, en été, vivre une quinzaine de jours au grand air, temps largement suffisant pour leur permettre d'être transportés à d'assez grandes distances.

En Islande, les agneaux, peu de temps après leur naissance, au printemps, sont séparés de leurs mères et emmenés paître dans les montagnes avec les autres moutons, tandis que les brebis sont maintenues aux environs des fermes, dans les pâturages voisins, où elles sont ordinairement gardées par des enfants de 10 à 14 ans. On les ramène à la ferme, matin et soir, pour la traite. Dans les endroits où elles pâturent, les brebis sont toujours accompagnées d'un chien. Il est évident que c'est surtout dans ces endroits que se trouvent les œufs de ténia échinocoque.

Mais la brebis n'avale pas tous les œufs. Un grand nombre de ceux-ci se fixent dans sa toison lorsqu'elle se couche sur le sol. Bref, elle ramasse des œufs de ténia au pâturage et les rapporte à la ferme. 
Arrivées là, les brebis sont rassemblées dans un parc, généralement si étroit que, serrées les unes contre les autres, elles ne peuvent bouger pendant qu'on les trait (fig.). La trayeuse s'accroupit au milieu du troupeau, les bêtes se frottant contre elle de tous côtés. Comme on le comprend, il y a grand danger que ses mains, ses vêtements, son visage soient souillés par les œufs retenus dans la laine des brebis. Dès lors, la route sera directe, non seulement jusqu'au tube digestif de la trayeuse elle-même, mais aussi jusqu'aux

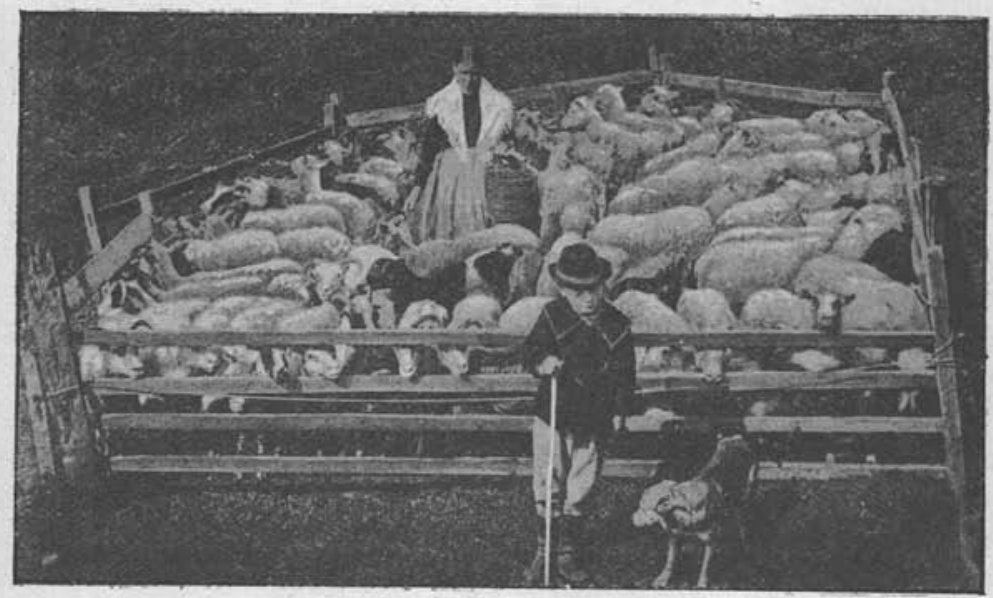

FIG. - La traite des brebis dans un parc en Islande.

aliments et boissons des autres personnes, si la propreté n'est pas suffisante.

La traite des brebis apparaît donc comme plus dangereuse à cet égard que ne l'est aucun autre métier en Islande. Naturellement, on peut supposer que les vaches, elles aussi, peuvent être souillées d'œufs de ténia, quoique à un moindre degré. La traite des vaches est également le rôle des femmes. Les hommes ne sont jamais en rapports aussi étroits avec les brebis laitières (les plus dangereuses) durant l'été. Ajoutons que ce sont surtout les femmes qui tondent les moutons, travaillent la laine sale et la lavent. Par suite, elles sont plus exposées à l'échinococcose que les hommes.

En Tunisie, l'ordre des choses est le même qu'en Islande : les femmes sont atteintes deux fois plus souvent que les hommes. J'ignore si les Arabes séparent les agneaux des brebis, comme chez nous. Je sais seulement qu'ils ne mangent pas les moutons adultes, mais seulement les agneaux (Dévé). Il m’apparaît vraisemblable 
qu'ils doivent traire les brebis dont ils sacrifient les agneaux et que cette traite doit être la fonction des femmes. On retrouverait donc la même cause qu'en Islande pour expliquer la différence de contamination des deux sexes. Les autres habitants de la Tunisie, les Français et les Juifs, qui ne s'occupent pas de l'élevage des animaux domestiques, sont bien quelquefois atteints de kystes hydatiques, mais les hommes autant que les femmes (Dévé).

En Australie et en Argentine, l'élevage du mouton a pour but la production de viande : d'où l'on pourrait conclure que les agneaux ne sont pas séparés de leurs mères. Et de là il résulte que les hommes s'occupent plus des moutons que les femmes.

F. Dévé dit que " le mouton ne joue aucun rôle direct dans la contamination de l'homme ". A la vérité, sa pensée était que la consommation de viande de mouton n'était pas dangereuse à ce point de vue (contrairement à l'opinion soutenue par le Prof. Lozano, de Saragosse). Son opinion est correcte jusque-là, mais pas plus loin.

Le mode principal de contamination humaine me semble être le suivant : Les œufs de ténia échinocoque se fixent dans la laine des brebis laitières et de là sont transmis aux femmes trayeuses, etc. Bien entendu, les œufs sont également transportés par le chien dans ses poils et non sur sa langue (Dévé). Mais, comparés aux moutons, les chiens sont si peu nombreux qu'ils ne doivent jouer qu'un rôle insignifiant. On peut supposer que des œufs se trouvent aussi dans le foin. Mais ces deux éléments étiologiques sont assez accessoires et d'ailleurs ils intéressent hommes et femmes au même degré. Le soin des moutons en hiver n'est guère dangereux ; car, par le froid et la gelée, les œufs se disséminent beaucoup moins ; de plus, leurs conditions vitales ne sont pas aussi favorables.

Dans son travail intitulé "Ekinokok-sygdommen "; Jonassen dit que Thorgrimur Johnsen (rapport de 1870) estimait douteux que le chien fût la seule source de l'échinococcose. Il en donnait pour preuve que son prédécesseur Skuli Thorarensen (médecin du canton de Rangarvallasysla, de 1834 à 1869) n'avait jamais vu un seul individu atteint de kyste hydatique dans le district de Bykkvibaer, quoique les habitants, pauvres et malpropres, aient des chiens comme les autres.

Au sujet de cette opinion, Jonassen écrit : "Ceci semble, en effet, très curieux. Mais un examen plus minutieux, loin de confirmer l'opinion de mon collègue, prouve le contraire. Bykkvibaer est un îlot baigné de tous côtés par le fleuve Bvera. Aujourd'hui (1882), il y a là 500 moutons, mais seulement durant l'hiver. Le nombre des habitants est de 270 seulement. Or, les chiens des districts où 
il y a peu de moutons ne sont pas aussi souvent porteurs de ténias qu'ailleurs. Dès lors, il est facile de comprendre qu'il y ait peu de malades atteints de kystes hydatiques dans un tel endroit. "

J'ai interrogé sur ce point mon collègue le $\mathrm{D}^{r} \mathrm{G}$. Gudfinnson qui, pendant douze ans, de 1911 à 1923 , fut médecin dudit canton. Il m'a dit exactement la même chose : il n'a jamais vu un individu atteint d'échinococcose dans Bykkvibaer. Voilà, certes, une singularité digne d'attention. Mais à chacun son opinion. Voici la mienne :

Aujourd'hui comme autrefois, Bykkvibaer est un ilot isolé. Sa population est de 300 habitants environ. Il n'y a pas beaucoup de moutons. Tous sont transportés hors de l'île au commencement du printemps, les brebis comme les autres. Jamais on n'y a séparé les agneaux de leurs mères. Ce n'est pas en accompagnant les moutons pendant l'été que les chiens s'infestent, mais seulement en automne, à l'époque de l'abattage. Aujourd'hui encore, on tue des moutons à Bykkvibaer, quoique en plus petit nombre qu'autrefois. Accompagnant leurs maîtres à la ville où les moutons sont vendus et abattus, les chiens ont l'occasion de trouver des "vessies d'eau ". Bref, il semble que la plupart des conditions nécessaires se trouvent réunies pour que les dits chiens s'infestent et contaminent les gens..... Seulement, les moutons ne sont pas réunis près des fermes, y rassemblant les œufs de ténia et les apportant aux habitants en été, saison qui offre les meilleures conditions vitales pour les œufs de ténias.

F. Dévé, dans un article sur "Les kystes hydatiques en Normandie ", se demande: "Pourquoi l'échinococcose, si rare en Basse-Normandie, est-elle relativement commune sur la rive droite de la Seine ? Il est possible que cette particularité soit en relation avec la persistance de l'élevage du mouton, demeuré plus développé sur le plateau cauchois que dans la plaine de Caen. Cependant d'autres raisons étiologiques et pathogéniques interviennent vraisemblablement. " On retrouve là les mêmes causes qu'à Bykkvibaer : moins de moutons porteurs d'œufs, moins d'échinococcose humaine, conformément à la loi qui veut que dans une région le nombre des gens atteints soit proportionnel à celui des moutons contaminés.

Les kystes hydatiques croissent lentement. Il-leur faut plusieurs années pour qu'ils donnent lieu à des troubles morbides. Or, il est de coutume, en Islande, que les filles commencent à traire les brebis de 10 à 14 ans environ. Si ce que nous avons dit du mode de contamination est exact, l'échinococcose devrait se montrer chez les 
femmes de 20 à 40 ans. Et c'est à cette période de l'existence que la différence entre le nombre des hommes et des femmes atteints par la maladie hydatique devrait être la plus grande.

C'est précisément ce qui a lieu, ainsi que Jonassen l'avait déjà indiqué et comme on pourra le voir par le tableau suivant que j'ai établi et où figurent le nombre, l'âge et le sexe des malades islandais, selon les observateurs :

\begin{tabular}{|c|c|c|c|c|c|}
\hline$A G E$ & J. Finsen & JONASSEN & G. MaGNuSSON & M EINARSSON & Total \\
\hline & H. F. & H. F. & H. & H. F. & H. F. \\
\hline $1-10$ ans. . & $10 \quad 10$ & 6 & 11 & 0 & $17 \quad 13$ \\
\hline $10-20$ ans . & $12 \quad 37$ & 54 & $\begin{array}{ll}3 & 10\end{array}$ & 3 & $23 \quad 52$ \\
\hline $20-40$ ans. & 2182 & 1122 & 2159 & 1338 & 66200 \\
\hline $40-60$ ans. & $21 \quad 34$ & $8 \quad 9$ & $26 \quad 37$ & $21 \quad 43$ & $76 \quad 123$ \\
\hline $\mathrm{Au}$-dessus de 60 ans & $\begin{array}{ll}9 & 9\end{array}$ & 0 & $\begin{array}{ll}3 & 8\end{array}$ & 7 & $19 \quad 24$ \\
\hline
\end{tabular}

Je ne doute pas que la contamination humaine ne se fasse de la manière que j'ai indiquée. Cette théorie résout à la fois la question de savoir pourquoi les femmes sont plus atteintes que les hommes, pourquoi c'est de 20 à 40 ans que la maladie se montre le plus, chez elles, et pourquoi la contamination des enfants est relativement rare.

$$
* *
$$

Au commencement de notre siècle, quelques fermiers islandais ont introduit cette nouveauté de ne plus séparer les agneaux des brebis, au printemps. Au contraire, agneaux et brebis ont été emmenés dans les montagnes avec les autres moutons. Le changement en question avait pour but d'engraisser les agneaux en les laissant têter tout l'été et d'en faire ainsi une meilleure marchandise. Cette coutume s'est répandue graduellement, au point que vers 1910 elle est devenue générale. Depuis lors, on ne voit plus un seul mouton près des fermes durant l'été : tous sont dans la montagne, loin de tout chien.

La nouveauté dont il s'agit devrait influer sur la contamination hydatique humaine, en diminuant singulièrement le danger, et aussi en réduisant la différence du danger entre les hommes et les femmes.

On pourrait se demander si, après ces vingt années écoulées, un certain effacement de la différence en question n'est pas déjà sen- 
sible. C'est ce que j'ai recherché en établissant le tableau suivant qui donne l'âge et le sexe des malades atteints de kystes hydatiques hospitalisés à Reykjavik de 1905 à 1924, inclusivement :

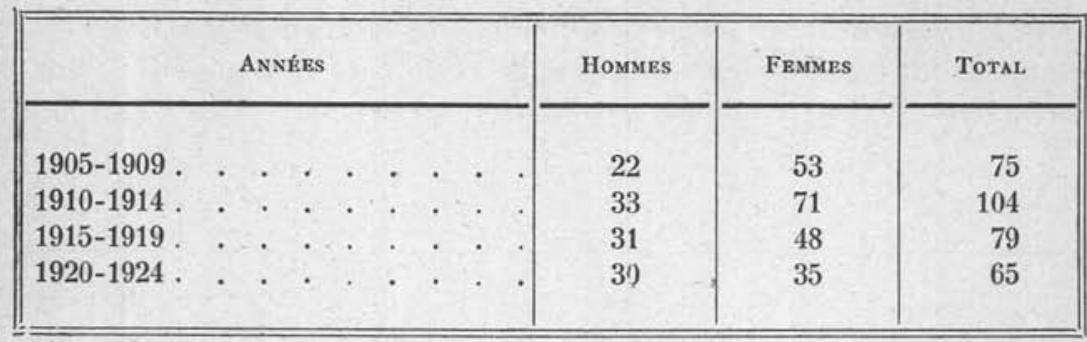

De ce tableau, il ressort avec évidence que la différence entre les malades des deux sexes va en s'atténuant. Quant à une diminution de l'échinococcose en général, elle n'apparaît pas ici nettement, bien que, en fait, la maladie diminue réellement de fréquence (1).

Je suppose que d'ici 5 ou 10 ans, les conséquences du nouveau mode d'élevage adopté par les fermiers seront plus évidentes encore. Sans doute, verra-t-on que la meilleure prophylaxie antihydatique appliquée en Islande jusqu'à ce jour, aura été le fait de ne plus traire les brebis, quoique cette modification ait eu, en principe, un autre but.

Par ce que je viens d'exposer, on peut voir que mon opinion sur la contamination échinococcique est bien différente de celle qui était admise jusqu'ici. Néanmoins, il n'y aura pas lieu de modifier les mesures prophylactiques. Celles-ci continueront d'être celles que Krabbe avait prescrites dès 1863: "Réduire le nombre des chiens. Ne pas les laisser avaler des "vessies d'eau ". Augmenter la propreté générale ".

Avant tout, il faut exhorter les gens à se laver les mains avant les repas (maladie des mains sales) et avant de servir le repas des autres. Les trayeuses devront prendre les plus grandes précautions, comme aussi ceux qui s'occupent de la laine sale, soit avant, soit après la tonte.

(1) Le nombre plus important des malades observés pendant la période 1910-1914 ne tient pas à une augmentation de fréquence de la maladie, mais au fait que c'est seulement à cette époque que les malades ont commencé à se confier au chirurgien plus qu'ils ne le faisaient autrefois. 